\title{
Growth and Yield of Sunflower as Influenced by Nutrient Management Practices
}

\author{
G. Ravishankar ${ }^{1 *}$, Lokanath H. Malligawad ${ }^{2}$ and B.K. Desai ${ }^{3}$ \\ ${ }^{1}$ ICAR-KVK, Hagari, Ballari, India \\ ${ }^{2}$ PGS, University of Agricultural Sciences, Dharwad, India \\ ${ }^{3}$ University of Agricultural Sciences, Raichur, India \\ *Corresponding author
}

\section{A B S T R A C T}

\section{Keywords}

Sunflower, Nutrient

Management,

Growth, Seed yield,

Oil yield and

Quality

Article Info

Accepted:

26 November 2019

Available Online:

10 December 2019

A field experiment was conducted during the year 2013 to study the effect of nutrient management practices on growth and yield of hybrid sunflower (cv. RSFH 130) in split plot design on medium deep black soil under protective irrigation at Agricultural Research Station, Hagari, Ballari, University of Agricultural Sciences, Raichur, Karnataka. Treatments consisted of four main plot treatments; different nutrient management practices through soil application viz., $\mathrm{M}_{1}$; application of RDF ( 90:90:60 $\left.\mathrm{kg} \mathrm{N}, \mathrm{P}_{2} \mathrm{O}_{5}, \mathrm{~K}_{2} \mathrm{O} / \mathrm{ha}\right), \mathrm{M}_{2}$; application of RDF + FYM @ $8 \mathrm{t} / \mathrm{ha}, \mathrm{M}_{3}$; application of $85 \% \mathrm{RDF}+\mathrm{FYM} @ 8 \mathrm{t} / \mathrm{ha}$ and $\mathrm{M}_{4}$; application of $60 \%$ $\mathrm{RDF}+\mathrm{FYM} @ 8 \mathrm{t} / \mathrm{ha}$ and four sub plot treatments; nutrient management practices through water soluble foliar grade fertilizers viz., $\mathrm{S}_{1}$; control, $\mathrm{S}_{2}$; foliar application of 19:19:19 water soluble foliar grade fertilizer @ 2\% at 25 and 40 DAS, $\mathrm{S}_{3}$; foliar application of 17:44 water soluble foliar grade fertilizer@ $2 \%$ at 25 and 40 DAS and $\mathrm{S}_{4}$; foliar application of urea @ 2\% at 25 and 40 DAS. Results revealed that application of RDF + FYM @ 8 t/ha along with foliar application of 19:19:19 water soluble foliar grade fertilizer@2\% at 25 and 40 DAS recorded higher plant height, stem girth, total dry matter production, head diameter, seed yield per plant, thousand seed weight, seed yield, stalk yield, oil content and oil yield of sunflower when compared to other treatment combinations.

\section{Introduction}

In India sunflower emerged as a promising potential oilseed crop in seventies because of its special characteristics viz., wider adaptability to varied climatic conditions, photo insensitivity, low seed rate, high yield potential, short duration, response to applied 
nutrients and high quality oil. In India sunflower is grown over an area of 0.55 million hectares with a production of 0.42 million tonnes and a productivity of $753 \mathrm{~kg} \mathrm{ha}^{-}$ 1 (Anon, 2016) which is far below than its potential. Karnataka accounts majority of the sunflower production ( 0.21 million tonnes) in India with an area of 0.36 million hectares and productivity of $597 \mathrm{~kg} \mathrm{ha}^{-1}$. The lower productivity of crop is mainly ascribed to cultivation of sunflower in less fertile marginal lands under low and uncertain rainfall situations with low and imbalanced use of fertilizers (Ramulu et al., 2011).

Sunflower is an important fast growing and high yielding energy rich oilseed crop which removes considerable amount of nutrients and ranks fourth next only to soybean, groundnut and rapeseed as a source of premier quality edible oil in the world. Unless the soils are replenished with all the nutrients taken up by the crop, there will be persistent nutrient exhaustion posing a great threat to sustainable production and in turn puts heavy demand on soil and applied nutrients (Hegde, and Sudhakarababu, 2009). Sunflower has a high nitrogen requirement that must be supplied throughout its growth and a shortage of nitrogen prior to flower initiation reduces the yield. Phosphorus is the second major nutrient after nitrogen in limiting the sunflower production as phosphorus is more prone for fixation rendering it as non available to plants due to many soil reactions and interactions with other elements. Further, Biradar et al., (2012) indicated wide variation in the current nutrient management practices and nutrient levels for obtaining potential yields. The research efforts made in respect to its nutritional requirements are scanty and imbalanced use of fertilizers has been identified as one of the critical constraints in sunflower production. Farmyard manure/organic manures combined with inorganic fertilizer management resulted in a higher increase in sunflower yield, Therefore, nutrient management in sunflower assumes importance for increasing productivity. Thus, an experiment was conducted to study the effect of different nutrient management practices on growth, yield and quality of sunflower.

\section{Materials and Methods}

Field experiment was conducted during post rainy season of 2013 at Agricultural Research Station, Hagari, Ballari which is situated in the Northern Dry Zone (Zone-3) of agro-climatic zones of Karnataka. The soil was medium black clayey in texture, low in organic carbon $(0.48 \%)$, low in available nitrogen $(191.0 \mathrm{~kg} \mathrm{~N}$ $\left.\mathrm{ha}^{-1}\right)$, medium in available phosphorus (34.30 $\mathrm{kg} \mathrm{P}_{2} \mathrm{O}_{5} \mathrm{ha}^{-1}$ ) and high in available potassium (480.0 $\mathrm{kg} \mathrm{K}_{2} \mathrm{O} \mathrm{ha}^{-1}$ ) with $\mathrm{pH}$ of 8.2. Sunflower hybrid RSFH-130, with duration of 100 days was used in the trial. The experiment was laid out in split plot design with three replications with a gross plot size of $21.60 \mathrm{~m}^{2}$ and grown with common cultivation practices. The experiment consisted of four main plot treatments viz., $\mathrm{M}_{1}$; application of $\mathrm{RDF}$ (90:90:60 $\left.\mathrm{kg} \quad \mathrm{N}, \quad \mathrm{P}_{2} \mathrm{O}_{5}, \quad \mathrm{~K}_{2} \mathrm{O} \quad / \mathrm{ha}\right), \mathrm{M}_{2}$; application of RDF + FYM @ 8 t/ha, $\mathrm{M}_{3}$; application of $85 \%$ RDF + FYM @ $8 \mathrm{t} / \mathrm{ha}$ and $\mathrm{M}_{4}$; application of $60 \% \mathrm{RDF}+\mathrm{FYM} @ 8$ t/ha and four sub plot treatments viz., $\mathrm{S}_{1}$; Control, $\mathrm{S}_{2}$; foliar application of 19:19:19 water soluble foliar grade fertilizer @ 2\% at 25 and 40 DAS, $\mathrm{S}_{3}$; foliar application of 17:44 water soluble foliar grade fertilizer @ 2\% at 25 and 40 DAS and $\mathrm{S}_{4}$; foliar application of urea @ 2\% at 25 and 40 DAS. The pretreated seeds with Imidacloprid and Ridomyl $\mathrm{MZ}$ were dibbled at a spacing of $60 \times 30 \mathrm{~cm}$. The fertilizer nitrogen, phosphorus and potassium were applied in the form of urea, di-ammonium phosphate and muriate of potash, respectively. Seventy five per cent of nitrogen and entire quantity of phosphorus and potassium fertilizers were applied as per the treatments at 
the time of sowing. Top dressing (in band placement) of remaining twenty five per cent of nitrogen in the form of urea was applied at 40 days after sowing (DAS). The experimental plots were kept weed free by integrated weed management practices involving preemergence herbicide application of Pendimethalin 30 EC @ 4.51 ha $^{-1}$ with two inter cultivations (25 and 40 DAS) and one hand weeding at 40 DAS. The crop was kept disease and insect free by appropriate plant protection measures. Periodic observations on growth attributes were recorded at 25, 50, 75 DAS, at harvest and yield attributes were recorded at harvest. Oil content was estimated through Nuclear Magnetic Resonance (NMR) spectrometer against standard reference sample. Data collected at different stages were subjected to statistical analysis as described by Gomez and Gomez (1984). Treatment means were compared using Duncan's Multiple Range Test (DMRT).

\section{Results and Discussion}

Effect of different nutrient management practices on growth and yield attributes of sunflower

It is evident from the various nutrient management studies that crop yield strongly depends on the supply of nutrients and among the fertilizer nutrients, nitrogen $(\mathrm{N})$ is critical, as it is a component of proteins, enzymes, nucleic acids and chlorophyll. Increased application rates of nitrogen leads to rapid leaf area development, prolongs life of foliage, increases leaf area duration after flowering and enhances crop assimilation consequently contributing to higher yield. Sunflower is an important fast growing and high yielding oilseed crop which removes considerable amount of nutrients to the extent of $63.3 \mathrm{~kg} \mathrm{~N}$, $19.1 \mathrm{~kg} \mathrm{P}_{2} \mathrm{O}_{5}$ and $126.0 \mathrm{~kg} \mathrm{~K}_{2} \mathrm{O}$ to produce one ton (Hegde and Sudhakarababu, 2009). Nitrogen requirement for sunflower crop is high and that must be supplied throughout its growth and any shortage prior to flowering results in reduced head diameter, number of seeds head ${ }^{-1}$, seed and oil yield. Phosphorus is the next major nutrient after nitrogen in limiting the sunflower production as phosphorus is more prone for fixation rendering it as unavailable to plants due to many soil reactions and interactions with other elements. Phosphorus is vital for plant growth and involved in energy transfer, photosynthesis, transformation of sugars, starches and nutrient movement within the plant. Adequate phosphorus supply results in better root penetration and proliferation contributing to production of photosynthates and their translocation to sink. When phosphorus is limiting the most striking effects is reduction in leaf expansion, leaf surface area and number of leaves (Hemalatha et al., 2013).

Experimental results revealed that among the main plot treatments (nutrient management through soil application) application of RDF (90:90:60 kg N, $\mathrm{P}_{2} \mathrm{O}_{5}, \mathrm{~K}_{2} \mathrm{O} \mathrm{ha}^{-1}$ ) + FYM @ $8 \mathrm{t}$ $\mathrm{ha}^{-1}$ recorded significantly higher plant height $(173.89 \mathrm{~cm})$, stem girth $(6.68 \mathrm{~cm})$, leaf area $\left(64.59 \mathrm{dm}^{2} /\right.$ plant $)$, leaf area duration $(81.72$ days), total dry matter production/plant (127.57 g/plant), head diameter $(15.77 \mathrm{~cm})$, seed yield/plant (43.40 g) and thousand seed weight $(57.85 \mathrm{~g})$ when compared to application of 60 per cent RDF + FYM @ $8 \mathrm{t}$ $\mathrm{ha}^{-1+}$ and application of RDF alone. Among the sub plot treatments (nutrient management through foliar application of water soluble fertilizers) foliar application of 19:19:19 N, $\mathrm{P}_{2} \mathrm{O}_{5}, \mathrm{~K}_{2} \mathrm{O}$ fertilizer @ $2 \%$ at 25 and 40 DAS produced higher plant height $(168.60 \mathrm{~cm})$, stem girth $(6.46 \mathrm{~cm})$, leaf area (60.69 $\mathrm{dm}^{2} /$ plant), leaf area duration (77.02 days), total dry matter production/plant (119.16 $\mathrm{g} /$ plant $)$, head diameter $(15.18 \mathrm{~cm})$, seed yield/plant (40.29 g) and thousand seed weight $(55.51 \mathrm{~g})$ when compared to control and foliar 
application of urea@2\% at 25 and 40 DAS (Table 1-4). Interaction of nutrient management through soil and foliar application indicated that application of RDF (90:90:60 kg N, $\left.\mathrm{P}_{2} \mathrm{O}_{5}, \mathrm{~K}_{2} \mathrm{Oha}^{-1}\right)+\mathrm{FYM} @ 8 \mathrm{t}$ $\mathrm{ha}^{-1}$ with foliar application of 19:19:19 N, $\mathrm{P}_{2} \mathrm{O}_{5}, \mathrm{~K}_{2} \mathrm{O}$ fertilizer @ $2 \%$ at 25 and 40 DAS produced higher plant height $(181.50 \mathrm{~cm})$, stem girth $(7.57 \mathrm{~cm})$, leaf area (70.97, $\mathrm{dm}^{2} /$ plant), higher leaf area duration (88.96 days), total dry matter production (136.97 g/plant), head diameter $(16.50 \mathrm{~cm})$, seed yield/plant $(48.10 \mathrm{~g})$ and thousand seed weight $(63.80 \mathrm{~g})$ when compared to other treatment combinations. However it remained on par with application of 85 per cent RDF + FYM @ $8 \mathrm{t} \mathrm{ha}^{-1}$ along with application of 19:19:19 water soluble foliar grade $\mathrm{N}, \mathrm{P}_{2} \mathrm{O}_{5}$, $\mathrm{K}_{2} \mathrm{O}$ fertilizer and 17:44 water soluble $\mathrm{N}$, $\mathrm{P}_{2} \mathrm{O}_{5}$, fertilizer treatment.

Improvement in yield attributing parameters could be attributed to better growth parameters in addition to total dry matter production at different stages of the crop growth and it's partitioning into different parts and also might be due to higher NPK levels supplied along with FYM and additional amount of nitrogen, phosphorus, potassium supplied through the foliar application at $25 \mathrm{DAS}$ and 40 DAS. Increased rates of nitrogen and phosphorus application leads to more rapid leaf area development, higher number of leaves, prolongs life of foliage, increases leaf area duration after flowering and enhances crop assimilation consequently contributing to higher yield.

These results are in conformity with the findings of Iqbal et al., (2008), Khaliq et al., (2008), Khaliq et al., (2009), Ramulu et al., (2011), Hemalatha et al., (2013), Banerjee et al., (2014), Jebarathnam and Sumalatha (2014), Gebremedhin et al., 2015 where they observed larger head size, higher seed yield/ plant, 1000 seed weight, higher total dry matter production, higher number of seeds/head with application of RDF along with organics and foliar application of nutrients in addition to soil application.

Effect of different nutrient management practices on seed yield, oil content and oil yield of sunflower

Results of the experiment indicated that among the main plot treatments (nutrient management through soil application) application of $\mathrm{RDF}$ (90:90:60 kg N, $\mathrm{P}_{2} \mathrm{O}_{5}$, $\left.\mathrm{K}_{2} \mathrm{O} \mathrm{ha}^{-1}\right)+\mathrm{FYM} @ 8 \mathrm{t} \mathrm{ha}^{-1}$ recorded significantly higher seed yield $(2403 \mathrm{~kg} / \mathrm{ha})$, stalk yield $(4530 \mathrm{~kg} / \mathrm{ha})$, oil content $(40.57 \%)$ and oil yield $(979 \mathrm{~kg} / \mathrm{ha})$ when compared to application of 60 per cent RDF + FYM @ $8 \mathrm{t}$ $\mathrm{ha}^{-1+}$ and application of RDF alone. Among the sub plot treatments (nutrient management through water soluble foliar grade fertilizers) foliar application of 19:19:19 N, $\mathrm{P}_{2} \mathrm{O}_{5}, \mathrm{~K}_{2} \mathrm{O}$ fertilizer@2\% at 25 and 40 DAS produced higher seed yield $(2233 \mathrm{~kg} / \mathrm{ha})$, stalk yield (4355 kg/ha), oil content (40.18\%) and oil yield $(905 \mathrm{~kg} / \mathrm{ha})$ when compared to control and foliar application of urea @ 2\% at 25 and 40 DAS (Table 5-6). Interaction revealed that application of $\mathrm{RDF}$ (90:90:60 kg N, $\mathrm{P}_{2} \mathrm{O}_{5}$, $\left.\mathrm{K}_{2} \mathrm{O} \mathrm{ha}^{-1}\right)+$ FYM @ $8 \mathrm{t} \mathrm{ha}^{-1}$ with foliar application of 19:19:19 N, $\mathrm{P}_{2} \mathrm{O}_{5}, \mathrm{~K}_{2} \mathrm{O}$ fertilizer @ $2 \%$ at 25 and 40 DAS recorded significantly higher seed yield $\left(2657 \mathrm{~kg} \mathrm{ha}^{-1}\right)$, stalk yield (4687 kg/ha), oil content $(43.07 \%)$ and oil yield (1144 kg/ha) when compared to other treatment combinations and remained on par with application of FYM @ $8 \mathrm{tha}^{-1}$ with 85 per cent RDF along with application of 19:19:19 water soluble foliar grade $\mathrm{N}, \mathrm{P}_{2} \mathrm{O}_{5}$, $\mathrm{K}_{2} \mathrm{O}$ fertilizer and 17:44 water soluble $\mathrm{N}, \mathrm{P}_{2} \mathrm{O}_{5}$ fertilizer treatment combinations. Increased seed yield, oil content and oil yield of sunflower could be directly attributed to response of crop to application of higher level of nutrients through soil and foliar application resulting in better morphological, growth and yield attributing characters. 
Table.1 Plant height and stem girth of sunflower at harvest as influenced by nutrient management practices through soil application and water soluble foliar fertilizers

\begin{tabular}{|c|c|c|c|c|c|c|c|c|c|c|c|}
\hline \multicolumn{2}{|r|}{ Nutrient management through soil application (NMPS) } & \multicolumn{10}{|c|}{ Nutrient management through water soluble foliar fertilizers (WSFF) } \\
\hline & & \multicolumn{5}{|c|}{ Plant height $(\mathrm{cm})$ at harvest } & \multicolumn{5}{|c|}{ Stem girth $(\mathrm{cm})$ at harvest } \\
\hline & & $\mathrm{WSFF}_{0}$ & \multirow{2}{*}{$\frac{\text { WSFF }_{1}}{165.57 \mathrm{~b}-\mathrm{f}}$} & $\mathrm{WSFF}_{2}$ & $\mathrm{WSFF}_{3}$ & Mean & $\mathrm{WSFF}_{0}$ & $\mathrm{WSFF}_{1}$ & $\mathrm{WSFF}_{2}$ & $\mathrm{WSFF}_{3}$ & Mean \\
\hline $\mathrm{NMPS}_{1}$ & $\begin{array}{l}\text { Application of recommended dose of fertilizer }(90 \mathrm{~kg} \mathrm{~N}, 90 \mathrm{~kg} \\
\mathrm{P}_{2} \mathrm{O}_{5} \text { and } 60 \mathrm{~kg} \mathrm{~K} \mathrm{k}_{2} \mathrm{O} / \mathrm{ha} \text { ) }\end{array}$ & $139.90 \mathrm{hi}$ & & $163.00 \mathrm{c}-\mathrm{f}$ & $159.70 \mathrm{~d}-\mathrm{g}$ & $157.04 \mathrm{~b}$ & $4.87 \mathrm{fg}$ & $5.93 \mathrm{bc}$ & $5.77 \mathrm{~b}-\mathrm{d}$ & $5.30 \mathrm{~d}-\mathrm{f}$ & $5.47 \mathrm{~b}$ \\
\hline $\mathrm{NMPS}_{2}$ & $\begin{array}{l}\text { Application of recommended dose of farmyard manure }(8.0 \\
\text { t/ha) and recommended dose of fertilizers }\left(90 \mathrm{~kg} \mathrm{~N}, 90 \mathrm{~kg} \mathrm{P}_{2} \mathrm{O}_{5}\right. \\
\text { and } 60 \mathrm{~kg} \mathrm{~K} 2 \mathrm{O} / \mathrm{ha})\end{array}$ & $161.53 \mathrm{c}-\mathrm{f}$ & $181.50 \mathrm{a}$ & $178.40 \mathrm{ab}$ & $174.13 \mathrm{a}-\mathrm{d}$ & 173.89 a & $5.47 \mathrm{c}-\mathrm{e}$ & $7.57 \mathrm{a}$ & $7.47 \mathrm{a}$ & $6.20 \mathrm{~b}$ & $6.68 \mathrm{a}$ \\
\hline $\mathrm{NMPS}_{3}$ & $\begin{array}{l}\text { Application of recommended dose of farmyard manure }(8.0 \\
\text { t/ha) and } 85 \% \text { of recommended dose of fertilizers }(76.5 \mathrm{~kg} \mathrm{~N} \text {, } \\
\left.76.5 \mathrm{~kg} \mathrm{P}_{2} \mathrm{O}_{5} \text { and } 51 \mathrm{~kg} \mathrm{~K}_{2} \mathrm{O} / \mathrm{ha}\right)\end{array}$ & $159.00 \mathrm{e}-\mathrm{g}$ & $176.03 \mathrm{a}-\mathrm{c}$ & 171.93 a-e & $166.17 \mathrm{~b}-\mathrm{e}$ & $168.28 \mathrm{ab}$ & $5.10 \mathrm{e}-\mathrm{g}$ & $7.27 \mathrm{a}$ & $7.13 \mathrm{a}$ & $5.87 \mathrm{bc}$ & $6.34 \mathrm{a}$ \\
\hline $\mathrm{NMPS}_{4}$ & $\begin{array}{l}\text { Application of recommended dose of farmyard manure (8.0 } \\
\text { t/ha) and } 60 \% \text { of recommended dose of fertilizers }(54 \mathrm{~kg} \mathrm{~N}, 54 \\
\left.\mathrm{kg} \mathrm{P}_{2} \mathrm{O}_{5} \text { and } 36 \mathrm{~kg} \mathrm{~K} \mathrm{~K}_{2} \mathrm{O} / \mathrm{ha}\right)\end{array}$ & $135.13 \mathrm{i}$ & $151.30 \mathrm{f}-\mathrm{h}$ & $146.90 \mathrm{~g}-\mathrm{i}$ & $142.73 \mathrm{hi}$ & $144.02 \mathrm{c}$ & $4.57 \mathrm{~g}$ & $5.07 \mathrm{e}-\mathrm{g}$ & $4.90 \mathrm{fg}$ & $4.67 \mathrm{~g}$ & $4.80 \mathrm{c}$ \\
\hline & Mean & $148.89 \mathrm{c}$ & 168.60 a & $\begin{array}{l}165.06 \\
\text { ab }\end{array}$ & $160.68 \mathrm{~b}$ & & $5.00 \mathrm{c}$ & $6.46 \mathrm{a}$ & $6.32 \mathrm{a}$ & $5.51 \mathrm{~b}$ & \\
\hline \multicolumn{2}{|c|}{ Comparing the means of } & \multicolumn{5}{|c|}{ S.Em. \pm} & \multicolumn{5}{|c|}{ S.Em. \pm} \\
\hline \multicolumn{2}{|c|}{ Nutrient management through soil application (NMPS) } & \multicolumn{5}{|c|}{3.46} & \multicolumn{5}{|c|}{0.12} \\
\hline \multicolumn{2}{|c|}{ Nutrient management through water soluble foliar fertilizers (WSFF) } & \multicolumn{5}{|c|}{2.22} & \multicolumn{5}{|c|}{0.08} \\
\hline \multicolumn{2}{|c|}{ WSFF at same NMPS } & \multicolumn{5}{|c|}{4.43} & \multicolumn{5}{|c|}{0.16} \\
\hline \multicolumn{2}{|c|}{ NMPS at same or different WSFF } & \multicolumn{5}{|c|}{5.16} & \multicolumn{5}{|c|}{0.19} \\
\hline \multicolumn{2}{|c|}{$\mathrm{WSFF}_{0}-$ Control (No foliar application of water soluble foliar fertilizer) } & & & \multicolumn{8}{|c|}{$\begin{array}{l}\mathrm{WSFF}_{1}-\mathrm{Foliar} \text { application of water soluble foliar 19:19:19 fertilizer }\left(19 \% \mathrm{~N}, 19 \% \mathrm{P}_{2} \mathrm{O}_{5}\right. \\
\left.\text { and } 19 \% \mathrm{~K}_{2} \mathrm{O}\right) @ 2 \% \text { at } 25 \text { and } 40 \text { days after sowing }\end{array}$} \\
\hline \multicolumn{2}{|c|}{$\begin{array}{l}\text { WSFF }_{2}-\text { Foliar application of water soluble foliar 17:44 fertilizer } \\
\qquad\left(17 \% \mathrm{~N} \text { and } 44 \% \mathrm{P}_{2} \mathrm{O}_{5}\right) @ 2 \% \text { at } 25 \text { and } 40 \text { days after sowing }\end{array}$} & & & \multicolumn{8}{|c|}{$\mathrm{WSFF}_{3}-$ Foliar application of urea fertilizer (46\% N) @ $2 \%$ at 25 and 40 days after sowing } \\
\hline \multicolumn{2}{|c|}{ DAS - Days after sowing } & & & \multicolumn{8}{|c|}{ NS - Non-significant } \\
\hline
\end{tabular}


Table.2 Leaf area per plant at 50 DAS and leaf area duration at 50-75 DAS of sunflower as influenced by nutrient management practices through soil application and water soluble foliar fertilizers

\begin{tabular}{|c|c|c|c|c|c|c|c|c|c|c|c|}
\hline \multicolumn{2}{|r|}{ Nutrient management through soil application (NMPS) } & \multicolumn{10}{|c|}{ Nutrient management through water soluble foliar fertilizers (WSFF) } \\
\hline & & \multicolumn{5}{|c|}{ Leaf area per plant $\left(\mathrm{dm}^{2}\right)$ at 50 DAS } & \multicolumn{5}{|c|}{ Leaf area duration (days) at $50-75$ DAS } \\
\hline & & WSFF $_{0}$ & \multirow{2}{*}{$\frac{\mathbf{W S F F}_{\mathbf{1}}}{53.40 \mathrm{c}}$} & $\mathrm{WSFF}_{2}$ & $\mathrm{WSFF}_{3}$ & Mean & $\mathrm{WSFF}_{\mathbf{0}}$ & $\mathrm{WSFF}_{1}$ & $\mathrm{WSFF}_{2}$ & $\mathrm{WSFF}_{3}$ & Mean \\
\hline $\mathrm{NMPS}_{1}$ & $\begin{array}{l}\text { Application of recommended dose of fertilizer }(90 \mathrm{~kg} \mathrm{~N}, 90 \mathrm{~kg} \\
\left.\mathrm{P}_{2} \mathrm{O}_{5} \text { and } 60 \mathrm{~kg} \mathrm{~K} \mathrm{~K}_{2} \mathrm{O} / \mathrm{ha}\right)\end{array}$ & $43.77 \mathrm{de}$ & & $51.63 \mathrm{c}$ & $49.67 \mathrm{~cd}$ & $49.62 \mathrm{~b}$ & $58.96 \mathrm{gh}$ & $69.62 \mathrm{~d}$ & $67.17 \mathrm{de}$ & $65.04 \mathrm{~d}-\mathrm{f}$ & $65.20 \mathrm{~b}$ \\
\hline $\mathrm{NMPS}_{2}$ & 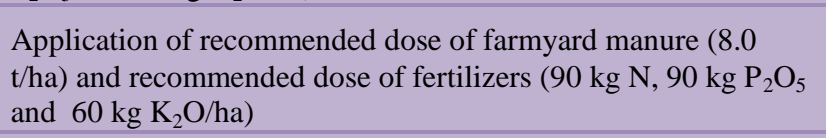 & $53.63 \mathrm{c}$ & 70.97 a & $68.53 \mathrm{ab}$ & $65.23 \mathrm{ab}$ & 64.59 a & $70.08 \mathrm{~d}$ & 88.96 a & $85.92 \mathrm{ab}$ & $81.92 \mathrm{bc}$ & $81.72 \mathrm{a}$ \\
\hline $\mathrm{NMPS}_{3}$ & $\begin{array}{l}\text { Application of recommended dose of farmyard manure }(8.0 \\
\text { t/ha) and } 85 \% \text { of recommended dose of fertilizers }(76.5 \mathrm{~kg} \mathrm{~N} \text {, } \\
\left.76.5 \mathrm{~kg} \mathrm{P}_{2} \mathrm{O}_{5} \text { and } 51 \mathrm{~kg} \mathrm{~K}_{2} \mathrm{O} / \mathrm{ha}\right)\end{array}$ & $52.77 \mathrm{c}$ & $69.20 \mathrm{a}$ & $66.20 \mathrm{ab}$ & $62.73 \mathrm{~b}$ & $62.73 \mathrm{a}$ & $68.21 \mathrm{de}$ & $86.62 \mathrm{ab}$ & $83.37 \mathrm{bc}$ & $78.25 \mathrm{c}$ & $79.11 \mathrm{a}$ \\
\hline $\mathrm{NMPS}_{4}$ & $\begin{array}{l}\text { Application of recommended dose of farmyard manure ( } 8.0 \\
\text { t/ha) and } 60 \% \text { of recommended dose of fertilizers }(54 \mathrm{~kg} \mathrm{~N}, 54 \\
\left.\mathrm{kg} \mathrm{P}_{2} \mathrm{O}_{5} \text { and } 36 \mathrm{~kg} \mathrm{~K}_{2} \mathrm{O} / \mathrm{ha}\right)\end{array}$ & $41.17 \mathrm{e}$ & $49.20 \mathrm{~cd}$ & $47.67 \mathrm{~cd}$ & $45.33 \mathrm{de}$ & $45.84 \mathrm{c}$ & $54.00 \mathrm{~h}$ & $62.87 \mathrm{e}-\mathrm{g}$ & $60.50 \mathrm{fg}$ & $57.92 \mathrm{gh}$ & $58.82 \mathrm{c}$ \\
\hline \multicolumn{2}{|r|}{ Mean } & $47.83 \mathrm{c}$ & 60.69 a & 58.51 ab & $55.74 \mathrm{~b}$ & & 62.81d & $77.02 a$ & $74.24 b$ & $70.78 c$ & \\
\hline \multicolumn{2}{|c|}{ Comparing the means of } & \multicolumn{5}{|c|}{ S.Em. \pm} & \multicolumn{5}{|c|}{ S.Em. \pm} \\
\hline \multicolumn{2}{|c|}{ Nutrient management through soil application (NMPS) } & \multicolumn{5}{|c|}{0.81} & \multicolumn{5}{|c|}{1.39} \\
\hline \multicolumn{2}{|c|}{ Nutrient management through water soluble foliar fertilizers (WSFF) } & \multicolumn{5}{|c|}{0.96} & \multicolumn{5}{|c|}{0.85} \\
\hline \multicolumn{2}{|c|}{ WSFF at same NMPS } & \multicolumn{5}{|c|}{1.91} & \multicolumn{5}{|c|}{1.70} \\
\hline \multicolumn{2}{|c|}{ NMPS at same or different WSFF } & \multicolumn{5}{|c|}{1.89} & \multicolumn{5}{|c|}{2.03} \\
\hline \multicolumn{4}{|c|}{$\mathrm{WSFF}_{0}-$ Control (No foliar application of water soluble foliar fertilizer) } & \multicolumn{8}{|c|}{$\begin{array}{l}\mathrm{WSFF}_{1}-\text { Foliar application of water soluble foliar 19:19:19 fertilizer }\left(19 \% \mathrm{~N}, 19 \% \mathrm{P}_{2} \mathrm{O}_{5}\right. \\
\left.\text { and } 19 \% \mathrm{~K}_{2} \mathrm{O}\right) @ 2 \% \text { at } 25 \text { and } 40 \text { days after sowing }\end{array}$} \\
\hline \multicolumn{4}{|c|}{$\begin{array}{l}\text { WSFF }_{2}-\text { Foliar application of water soluble foliar 17:44 fertilizer } \\
\qquad\left(17 \% \mathrm{~N} \text { and } 44 \% \mathrm{P}_{2} \mathrm{O}_{5}\right) @ 2 \% \text { at } 25 \text { and } 40 \text { days after sowing }\end{array}$} & \multicolumn{8}{|c|}{$\mathrm{WSFF}_{3}-$ Foliar application of urea fertilizer (46\% N) @ $2 \%$ at 25 and 40 days after sowing } \\
\hline \multicolumn{4}{|c|}{ DAS - Days after sowing } & \multicolumn{8}{|c|}{ NS - Non-significant } \\
\hline
\end{tabular}


Table.3 Total dry matter production and head diameter of sunflower at harvest as influenced by nutrient management practices through soil application and water soluble foliar fertilizers

\begin{tabular}{|c|c|c|c|c|c|c|c|c|c|c|c|}
\hline \multicolumn{2}{|r|}{ Nutrient management through soil application (NMPS) } & \multicolumn{10}{|c|}{ Nutrient management through water soluble foliar fertilizers (WSFF) } \\
\hline & & \multicolumn{5}{|c|}{ Total dry matter production (g/plant) at harvest } & \multicolumn{5}{|c|}{ Head Diameter $(\mathrm{cm})$ at harvest } \\
\hline & & $\mathrm{WSFF}_{0}$ & \multirow{2}{*}{$\begin{array}{c}\text { WSFF }_{1} \\
112.77 \mathrm{de}\end{array}$} & \multirow{2}{*}{$\frac{\mathbf{W S F F}_{\mathbf{2}}}{107.30 \mathrm{e}}$} & \multirow{2}{*}{$\begin{array}{c}\mathbf{W S F F}_{\mathbf{3}} \\
103.43 \mathrm{ef}\end{array}$} & \multirow{2}{*}{ Mean } & \multirow{2}{*}{$\frac{\mathbf{W S F F}_{\mathbf{0}}}{12.40 \mathrm{~g}-\mathrm{i}}$} & \multirow{2}{*}{$\begin{array}{c}\text { WSFF }_{1} \\
14.90 \mathrm{~b}-\mathrm{e}\end{array}$} & \multirow{2}{*}{$\frac{\text { WSFF }_{2}}{14.53 \mathrm{c}-\mathrm{f}}$} & \multirow{2}{*}{$\begin{array}{c}\text { WSFF }_{3} \\
13.63 \mathrm{e}-\mathrm{g}\end{array}$} & \multirow{2}{*}{ Mean } \\
\hline $\mathrm{NMPS}_{1}$ & $\begin{array}{l}\text { Application of recommended dose of fertilizer }(90 \mathrm{~kg} \mathrm{~N}, 90 \mathrm{~kg} \\
\left.\mathrm{P}_{2} \mathrm{O}_{5} \text { and } 60 \mathrm{~kg} \mathrm{~K}_{2} \mathrm{O} / \mathrm{ha}\right)\end{array}$ & $90.30 \mathrm{gh}$ & & & & & & & & & \\
\hline $\mathrm{NMPS}_{2}$ & $\begin{array}{l}\text { Application of recommended dose of farmyard manure }(8.0 \\
\text { t/ha) and recommended dose of fertilizers }(90 \mathrm{~kg} \mathrm{~N}, 90 \mathrm{~kg} \\
\left.\mathrm{P}_{2} \mathrm{O}_{5} \text { and } 60 \mathrm{~kg} \mathrm{~K} 2 \mathrm{O} / \mathrm{ha}\right)\end{array}$ & $113.20 \mathrm{de}$ & $136.97 \mathrm{a}$ & $132.83 \mathrm{ab}$ & $\begin{array}{l}127.27 \mathrm{a}- \\
\mathrm{c}\end{array}$ & $127.57 \mathbf{a}$ & $14.60 \mathrm{c}-\mathrm{f}$ & $16.50 \mathrm{a}$ & $16.40 \mathrm{a}$ & $15.57 \mathrm{a}-\mathrm{d}$ & $15.77 \mathrm{a}$ \\
\hline $\mathrm{NMPS}_{3}$ & $\begin{array}{l}\text { Application of recommended dose of farmyard manure }(8.0 \\
\text { t/ha) and } 85 \% \text { of recommended dose of fertilizers }(76.5 \mathrm{~kg} \mathrm{~N} \text {, } \\
\left.76.5 \mathrm{~kg} \mathrm{P}_{2} \mathrm{O}_{5} \text { and } 51 \mathrm{~kg} \mathrm{~K}_{2} \mathrm{O} / \mathrm{ha}\right)\end{array}$ & $108.30 \mathrm{e}$ & $129.90 \mathrm{ab}$ & $125.03 \mathrm{bc}$ & $119.27 \mathrm{~cd}$ & $120.63 b$ & $14.30 \mathrm{~d}-\mathrm{f}$ & $16.10 \mathrm{ab}$ & $15.90 \mathrm{a}-\mathrm{c}$ & $15.20 \mathrm{a}-\mathrm{d}$ & $15.38 \mathrm{a}$ \\
\hline $\mathrm{NMPS}_{4}$ & $\begin{array}{l}\text { Application of recommended dose of farmyard manure }(8.0 \\
\text { t/ha) and } 60 \% \text { of recommended dose of fertilizers }(54 \mathrm{~kg} \mathrm{~N} \text {, } \\
\left.54 \mathrm{~kg} \mathrm{P}_{2} \mathrm{O}_{5} \text { and } 36 \mathrm{~kg} \mathrm{~K}_{2} \mathrm{O} / \mathrm{ha}\right)\end{array}$ & $85.30 \mathrm{~h}$ & $97.00 \mathrm{fg}$ & $93.17 \mathrm{gh}$ & $90.70 \mathrm{gh}$ & $91.54 \mathrm{~d}$ & $11.23 \mathrm{i}$ & $13.23 \mathrm{f}-\mathrm{h}$ & 12.70gh & 12.03hi & $12.30 \mathrm{c}$ \\
\hline \multicolumn{2}{|r|}{ Mean } & $99.28 \mathrm{c}$ & 119.16a & 114.58ab & $110.17 \mathrm{~b}$ & & $13.13 \mathrm{c}$ & 15.18a & 14.88a & 14.11b & \\
\hline \multicolumn{2}{|c|}{ Comparing the means of } & \multicolumn{5}{|c|}{ S.Em. \pm} & \multicolumn{5}{|c|}{ S.Em. \pm} \\
\hline \multicolumn{2}{|c|}{ Nutrient management through soil application (NMPS) } & \multicolumn{5}{|c|}{$1.34^{-}$} & \multicolumn{5}{|c|}{0.38} \\
\hline \multicolumn{2}{|c|}{ Nutrient management through water soluble foliar fertilizers (WSFF) } & \multicolumn{5}{|c|}{1.60} & \multicolumn{5}{|c|}{0.23} \\
\hline \multicolumn{2}{|c|}{ WSFF at same NMPS } & \multicolumn{5}{|c|}{3.20} & \multicolumn{5}{|c|}{0.46} \\
\hline \multicolumn{2}{|c|}{ NMPS at same or different WSFF } & \multicolumn{5}{|c|}{3.08} & \multicolumn{5}{|c|}{0.55} \\
\hline \multicolumn{4}{|c|}{$\mathrm{WSFF}_{0}-$ Control (No foliar application of water soluble foliar fertilizer) } & \multicolumn{8}{|c|}{$\begin{array}{l}\mathrm{WSFF}_{1}-\text { Foliar application of water soluble foliar 19:19:19 fertilizer }\left(19 \% \mathrm{~N}, 19 \% \mathrm{P}_{2} \mathrm{O}_{5}\right. \\
\left.\text { and } 19 \% \mathrm{~K}_{2} \mathrm{O}\right) @ 2 \% \text { at } 25 \text { and } 40 \text { days after sowing }\end{array}$} \\
\hline \multicolumn{4}{|c|}{$\begin{array}{l}\mathrm{WSFF}_{2}-\text { Foliar application of water soluble foliar 17:44 fertilizer } \\
\left(17 \% \mathrm{~N} \text { and } 44 \% \mathrm{P}_{2} \mathrm{O}_{5}\right) @ 2 \% \text { at } 25 \text { and } 40 \text { days after sowing }\end{array}$} & \multicolumn{8}{|c|}{$\mathrm{WSFF}_{3}-$ Foliar application of urea fertilizer (46\% N) @ $2 \%$ at 25 and 40 days after sowing } \\
\hline \multicolumn{4}{|c|}{ DAS - Days after sowing } & \multicolumn{8}{|c|}{ NS - Non-significant } \\
\hline
\end{tabular}


Table.4 Seed yield per plant and 1000 seed weight of sunflower as influenced by nutrient management practices through soil application and water soluble foliar fertilizers

\begin{tabular}{|c|c|c|c|c|c|c|c|c|c|c|c|}
\hline \multicolumn{2}{|r|}{ Nutrient management through soil application (NMPS) } & \multicolumn{10}{|c|}{ Nutrient management through water soluble foliar fertilizers (WSFF) } \\
\hline & & \multicolumn{5}{|c|}{ Seed yield per plant (g) } & \multicolumn{5}{|c|}{1000 seed weight $(\mathrm{g})$} \\
\hline & & WSFF $_{0}$ & \multirow{2}{*}{$\frac{\text { WSFF }_{\mathbf{1}}}{36.97 \mathrm{~b}-\mathrm{d}}$} & $\mathrm{WSFF}_{2}$ & $\mathrm{WSFF}_{3}$ & Mean & $\mathrm{WSFF}_{\mathbf{0}}$ & $\mathrm{WSFF}_{1}$ & $\mathrm{WSFF}_{2}$ & $\mathrm{WSFF}_{3}$ & Mean \\
\hline $\mathrm{NMPS}_{1}$ & $\begin{array}{l}\text { Application of recommended dose of fertilizer }(90 \mathrm{~kg} \mathrm{~N}, 90 \mathrm{~kg} \\
\left.\mathrm{P}_{2} \mathrm{O}_{5} \text { and } 60 \mathrm{~kg} \mathrm{~K} \mathrm{~K}_{2} \mathrm{O} / \mathrm{ha}\right)\end{array}$ & $27.33 \mathrm{e}-\mathrm{g}$ & & $36.23 \mathrm{~b}-\mathrm{e}$ & $33.60 \mathrm{c}-\mathrm{f}$ & 33.53b & $42.63 \mathrm{e}$ & $54.57 \mathrm{bc}$ & $51.20 \mathrm{c}$ & $49.70 \mathrm{~cd}$ & $49.53 b$ \\
\hline $\mathrm{NMPS}_{2}$ & $\begin{array}{l}\text { Application of recommended dose of farmyard manure }(8.0 \\
\text { t/ha) and recommended dose of fertilizers }\left(90 \mathrm{~kg} \mathrm{~N}, 90 \mathrm{~kg} \mathrm{P}_{2} \mathrm{O}_{5}\right. \\
\left.\text { and } 60 \mathrm{~kg} \mathrm{~K} \mathrm{~K}_{2} \mathrm{O} / \mathrm{ha}\right)\end{array}$ & $37.23 \mathrm{~b}-\mathrm{d}$ & $48.10 \mathrm{a}$ & 47.37 a & $40.88 \mathrm{a}-\mathrm{c}$ & 43.40 a & $51.10 \mathrm{c}$ & $63.80 \mathrm{a}$ & $62.37 \mathrm{a}$ & $54.13 \mathrm{bc}$ & $57.85 \mathrm{a}$ \\
\hline $\mathrm{NMPS}_{3}$ & $\begin{array}{l}\text { Application of recommended dose of farmyard manure }(8.0 \\
\text { t/ha) and } 85 \% \text { of recommended dose of fertilizers }(76.5 \mathrm{~kg} \mathrm{~N} \text {, } \\
\left.76.5 \mathrm{~kg} \mathrm{P}_{2} \mathrm{O}_{5} \text { and } 51 \mathrm{~kg} \mathrm{~K} \mathrm{~K}_{2} \mathrm{O} / \mathrm{ha}\right)\end{array}$ & $33.77 \mathrm{c}-\mathrm{f}$ & $44.90 \mathrm{ab}$ & $43.97 \mathrm{ab}$ & $37.20 \mathrm{~b}-\mathrm{d}$ & 39.96 a & $49.87 \mathrm{~cd}$ & $60.20 \mathrm{ab}$ & $59.57 \mathrm{ab}$ & $55.23 b c$ & $56.22 \mathrm{a}$ \\
\hline $\mathrm{NMPS}_{4}$ & $\begin{array}{l}\text { Application of recommended dose of farmyard manure ( } 8.0 \\
\mathrm{t} / \mathrm{ha} \text { ) and } 60 \% \text { of recommended dose of fertilizers ( } 54 \mathrm{~kg} \mathrm{~N}, 54 \\
\left.\mathrm{~kg} \mathrm{P}_{2} \mathrm{O}_{5} \text { and } 36 \mathrm{~kg} \mathrm{~K}_{2} \mathrm{O} / \mathrm{ha}\right)\end{array}$ & $22.00 \mathrm{~g}$ & $31.20 \mathrm{~d}-\mathrm{f}$ & $30.03 \mathrm{~d}-\mathrm{g}$ & $26.63 \mathrm{fg}$ & 27.47 c & $39.60 \mathrm{e}$ & $43.47 \mathrm{de}$ & $42.87 \mathrm{e}$ & $40.33 \mathrm{e}$ & $41.57 \mathrm{c}$ \\
\hline \multicolumn{2}{|r|}{ Mean } & $30.08 c$ & $40.29 a$ & $39.40 \mathrm{a}$ & $34.58 b$ & & $45.80 \mathrm{c}$ & 55.51a & $54.00 a$ & 49.85b & \\
\hline \multicolumn{2}{|c|}{ Comparing the means of } & \multicolumn{5}{|c|}{ S.Em. \pm} & \multicolumn{5}{|c|}{ S.Em. \pm} \\
\hline \multicolumn{2}{|c|}{ Nutrient management through soil application (NMPS) } & \multicolumn{5}{|c|}{$1.24^{-}$} & \multicolumn{5}{|c|}{1.57} \\
\hline \multicolumn{2}{|c|}{ Nutrient management through water soluble foliar fertilizers (WSFF) } & \multicolumn{5}{|c|}{1.41} & \multicolumn{5}{|c|}{1.09} \\
\hline \multicolumn{2}{|c|}{ WSFF at same NMPS } & \multicolumn{5}{|c|}{2.81} & \multicolumn{5}{|c|}{2.18} \\
\hline \multicolumn{2}{|c|}{ NMPS at same or different WSFF } & \multicolumn{5}{|c|}{2.73} & \multicolumn{5}{|c|}{2.45} \\
\hline \multicolumn{4}{|c|}{$\mathrm{WSFF}_{0}-$ Control (No foliar application of water soluble foliar fertilizer) } & \multicolumn{8}{|c|}{$\begin{array}{l}\text { WSFF }_{1}-\text { Foliar application of water soluble foliar 19:19:19 fertilizer }\left(19 \% \mathrm{~N}, 19 \% \mathrm{P}_{2} \mathrm{O}_{5}\right. \\
\left.\text { and } 19 \% \mathrm{~K}_{2} \mathrm{O}\right) @ 2 \% \text { at } 25 \text { and } 40 \text { days after sowing }\end{array}$} \\
\hline \multicolumn{4}{|c|}{$\begin{array}{l}\text { WSFF }_{2}-\text { Foliar application of water soluble foliar 17:44 fertilizer } \\
\qquad\left(17 \% \mathrm{~N} \text { and } 44 \% \mathrm{P}_{2} \mathrm{O}_{5}\right) @ 2 \% \text { at } 25 \text { and } 40 \text { days after sowing }\end{array}$} & \multicolumn{8}{|c|}{$\mathrm{WSFF}_{3}-$ Foliar application of urea fertilizer $(46 \% \mathrm{~N}) @ 2 \%$ at 25 and 40 days after sowing } \\
\hline \multicolumn{4}{|c|}{ DAS - Days after sowing } & \multicolumn{8}{|c|}{ NS - Non-significant } \\
\hline
\end{tabular}


Table.5 Seed yield and stalk yield of sunflower as influenced by nutrient management practices through soil application and water soluble foliar fertilizers

\begin{tabular}{|c|c|c|c|c|c|c|c|c|c|c|c|}
\hline \multicolumn{2}{|r|}{ Nutrient management through soil application (NMPS) } & \multicolumn{10}{|c|}{ Nutrient management through water soluble foliar fertilizers (WSFF) } \\
\hline & & \multicolumn{5}{|c|}{ Seed yield (kg/ha) } & \multicolumn{5}{|c|}{ Stalk yield (kg/ha) } \\
\hline & & $\mathrm{WSFF}_{0}$ & \multirow{2}{*}{$\frac{\mathbf{W S F F}_{1}}{2025 \mathrm{c}-\mathrm{e}}$} & $\mathrm{WSFF}_{2}$ & $\mathrm{WSFF}_{3}$ & Mean & $\mathrm{WSFF}_{0}$ & $\mathrm{WSFF}_{1}$ & $\mathrm{WSFF}_{2}$ & $\mathrm{WSFF}_{3}$ & Mean \\
\hline $\mathrm{NMPS}_{1}$ & $\begin{array}{l}\text { Application of recommended dose of fertilizer }(90 \mathrm{~kg} \mathrm{~N}, 90 \mathrm{~kg} \\
\left.\mathrm{P}_{2} \mathrm{O}_{5} \text { and } 60 \mathrm{~kg} \mathrm{~K}{ }_{2} \mathrm{O} / \mathrm{ha}\right)\end{array}$ & $1794 \mathrm{e}-\mathrm{g}$ & & 1921d-f & $1863 d-f$ & $1901 b$ & $3848 d-g$ & 4310a-e & $4270 a-e$ & 4040b-f & $4117 \mathrm{~b}$ \\
\hline $\mathrm{NMPS}_{2}$ & $\begin{array}{l}\text { Application of recommended dose of farmyard manure }(8.0 \\
\text { t/ha) and recommended dose of fertilizers }\left(90 \mathrm{~kg} \mathrm{~N}, 90 \mathrm{~kg} \mathrm{P}_{2} \mathrm{O}_{5}\right. \\
\left.\text { and } 60 \mathrm{~kg} \mathrm{~K} \mathrm{~K}_{2} \mathrm{O} / \mathrm{ha}\right)\end{array}$ & 2060c-e & $2657 \mathrm{a}$ & $2567 \mathrm{ab}$ & $2326 a-c$ & 2403a & $4293 a-e$ & $4687 a$ & $4620 \mathrm{ab}$ & $4520 \mathrm{ab}$ & $4530 \mathrm{a}$ \\
\hline $\mathrm{NMPS}_{3}$ & $\begin{array}{l}\text { Application of recommended dose of farmyard manure }(8.0 \\
\text { t/ha) and } 85 \% \text { of recommended dose of fertilizers }(76.5 \mathrm{~kg} \mathrm{~N} \text {, } \\
\left.76.5 \mathrm{~kg} \mathrm{P}_{2} \mathrm{O}_{5} \text { and } 51 \mathrm{~kg} \mathrm{~K} \mathrm{~K}_{2} \mathrm{O} / \mathrm{ha}\right)\end{array}$ & 1905d-f & $2581 \mathrm{ab}$ & $2403 a-c$ & $2222 b-d$ & 2278a & $4260 a-e$ & $4553 \mathrm{ab}$ & $4497 a-c$ & 4450 a-d & $4440 \mathrm{a}$ \\
\hline $\mathrm{NMPS}_{4}$ & 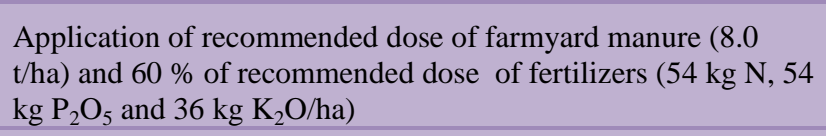 & $1454 \mathrm{~g}$ & $1667 \mathrm{e}-\mathrm{g}$ & $1597 \mathrm{fg}$ & $1551 \mathrm{fg}$ & $1567 \mathrm{c}$ & $3567 f$ & $3870 \mathrm{c}-\mathrm{f}$ & $3720 \mathrm{ef}$ & $3707 \mathrm{ef}$ & $3716 c$ \\
\hline \multicolumn{2}{|r|}{ Mean } & $1803 c$ & 2233a & 2122ab & 1991b & & 3992b & 4355a & 4277ab & 4179ab & \\
\hline \multicolumn{2}{|c|}{ Nutrient management through soil application (NMPS) } & \multicolumn{5}{|c|}{66} & \multicolumn{5}{|c|}{56} \\
\hline \multicolumn{2}{|c|}{ Nutrient management through water soluble foliar fertilizers (WSFF) } & \multicolumn{5}{|c|}{61} & \multicolumn{5}{|c|}{95} \\
\hline \multicolumn{2}{|c|}{ WSFF at same NMPS } & \multicolumn{5}{|c|}{121} & \multicolumn{5}{|c|}{189} \\
\hline \multicolumn{2}{|c|}{ NMPS at same or different WSFF } & \multicolumn{5}{|c|}{123} & \multicolumn{5}{|c|}{175} \\
\hline \multicolumn{4}{|c|}{$\mathrm{WSFF}_{0}-$ Control (No foliar application of water soluble foliar fertilizer) } & \multicolumn{8}{|c|}{ 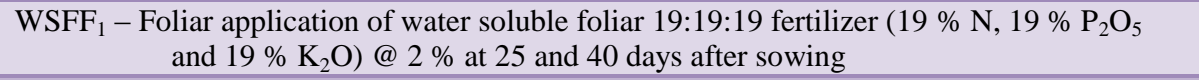 } \\
\hline \multicolumn{4}{|c|}{$\begin{array}{l}\text { WSFF }_{2}-\text { Foliar application of water soluble foliar 17:44 fertilizer } \\
\qquad\left(17 \% \mathrm{~N} \text { and } 44 \% \mathrm{P}_{2} \mathrm{O}_{5}\right) @ 2 \% \text { at } 25 \text { and } 40 \text { days after sowing }\end{array}$} & \multicolumn{8}{|c|}{$\mathrm{WSFF}_{3}-$ Foliar application of urea fertilizer $(46 \% \mathrm{~N}) @ 2 \%$ at 25 and 40 days after sowing } \\
\hline \multicolumn{4}{|c|}{ DAS - Days after sowing } & \multicolumn{8}{|c|}{ NS - Non-significant } \\
\hline
\end{tabular}


Table.6 Seed oil content and oil yield of sunflower as influenced by nutrient management practices through soil application and water soluble foliar fertilizers

\begin{tabular}{|c|c|c|c|c|c|c|c|c|c|c|c|}
\hline \multicolumn{2}{|r|}{ Nutrient management through soil application (NMPS) } & \multicolumn{10}{|c|}{ Nutrient management through water soluble foliar fertilizers (WSFF) } \\
\hline & & \multicolumn{5}{|c|}{ Seed oil content $(\%)$} & \multicolumn{5}{|c|}{ Oil yield (kg/ha) } \\
\hline & & \multirow{2}{*}{$\frac{\mathbf{W S F F}_{\mathbf{0}}}{35.17 \text { ef }}$} & \multirow{2}{*}{\begin{tabular}{|c|}
$\mathbf{W S F F}_{\mathbf{1}}$ \\
$38.77 \mathrm{a}-\mathrm{f}$
\end{tabular}} & \multirow{2}{*}{$\frac{\mathbf{W S F F}_{\mathbf{2}}}{37.83 \text { b-f }}$} & \multirow{2}{*}{$\frac{\mathbf{W S F F}_{\mathbf{3}}}{36.50 \mathrm{c}-\mathrm{f}}$} & \multirow{2}{*}{$\begin{array}{c}\text { Mean } \\
37.07 \text { b }\end{array}$} & \multirow{2}{*}{$\begin{array}{l}\text { WSFF }_{\mathbf{0}} \\
628 \mathrm{f}-\mathrm{h}\end{array}$} & \multirow{2}{*}{$\begin{array}{l}\text { WSFF }_{1} \\
788 \mathrm{~d}-\mathrm{f}\end{array}$} & \multirow{2}{*}{$\begin{array}{l}\text { WSFF }_{2} \\
721 \mathrm{e}-\mathrm{g}\end{array}$} & \multirow{2}{*}{$\begin{array}{l}\text { WSFF }_{3} \\
682 \mathrm{e}-\mathrm{g}\end{array}$} & \multirow{2}{*}{ Mean } \\
\hline $\mathrm{NMPS}_{1}$ & $\begin{array}{l}\text { Application of recommended dose of fertilizer }(90 \mathrm{~kg} \mathrm{~N}, 90 \mathrm{~kg} \\
\left.\mathrm{P}_{2} \mathrm{O}_{5} \text { and } 60 \mathrm{~kg} \mathrm{~K} \mathrm{~K}_{2} \mathrm{O} / \mathrm{ha}\right)\end{array}$ & & & & & & & & & & \\
\hline $\mathrm{NMPS}_{2}$ & $\begin{array}{l}\text { Application of recommended dose of farmyard manure }(8.0 \\
\text { t/ha) and recommended dose of fertilizers }\left(90 \mathrm{~kg} \mathrm{~N}, 90 \mathrm{~kg} \mathrm{P}_{2} \mathrm{O}_{5}\right. \\
\left.\text { and } 60 \mathrm{~kg} \mathrm{~K} \mathrm{~K}_{2} \mathrm{O} / \mathrm{ha}\right)\end{array}$ & $38.03 \mathrm{~b}-\mathrm{f}$ & $43.07 \mathrm{a}$ & $42.07 \mathrm{ab}$ & 39.10 a-e & 40.57 a & $780 \mathrm{~d}-\mathrm{f}$ & 1144 a & $1079 \mathrm{ab}$ & $913 \mathrm{~b}-\mathrm{d}$ & 979 a \\
\hline $\mathrm{NMPS}_{3}$ & $\begin{array}{l}\text { Application of recommended dose of farmyard manure }(8.0 \\
\text { t/ha) and } 85 \% \text { of recommended dose of fertilizers }(76.5 \mathrm{~kg} \mathrm{~N} \text {, } \\
\left.76.5 \mathrm{~kg} \mathrm{P}_{2} \mathrm{O}_{5} \text { and } 51 \mathrm{~kg} \mathrm{~K}_{2} \mathrm{O} / \mathrm{ha}\right)\end{array}$ & $37.40 \mathrm{~b}-\mathrm{f}$ & $41.07 \mathrm{a}-\mathrm{c}$ & $40.47 \mathrm{a}-\mathrm{d}$ & $38.47 \mathrm{~b}-\mathrm{f}$ & $39.35 \mathrm{a}$ & 708 e-g & $1057 \mathrm{ab}$ & $971 \mathrm{a}-\mathrm{c}$ & $860 \mathrm{c}-\mathrm{e}$ & 899 a \\
\hline $\mathrm{NMPS}_{4}$ & $\begin{array}{l}\text { Application of recommended dose of farmyard manure ( } 8.0 \\
\text { t/ha) and } 60 \% \text { of recommended dose of fertilizers ( } 54 \mathrm{~kg} \mathrm{~N}, 54 \\
\left.\mathrm{~kg} \mathrm{P}_{2} \mathrm{O}_{5} \text { and } 36 \mathrm{~kg} \mathrm{~K} \mathrm{~K}_{2} \mathrm{O} / \mathrm{ha}\right)\end{array}$ & $34.23 \mathrm{f}$ & $37.80 \mathrm{~b}-\mathrm{f}$ & $36.20 \mathrm{~d}-\mathrm{f}$ & $35.77 \mathrm{~d}-\mathrm{f}$ & $36.00 \mathrm{~b}$ & $495 \mathrm{~h}$ & $632 \mathrm{f}-\mathrm{h}$ & $583 \mathrm{gh}$ & $558 \mathrm{gh}$ & $567 \mathrm{c}$ \\
\hline & Mean & $36.21 \mathrm{c}$ & 40.18a & 39.14ab & 37.46bc & & $653 \mathrm{c}$ & $905 a$ & 838a & 753b & \\
\hline \multicolumn{2}{|c|}{ Comparing the means of } & \multicolumn{5}{|c|}{ S.Em. \pm} & \multicolumn{5}{|c|}{ S.Em. \pm} \\
\hline \multicolumn{2}{|c|}{ Nutrient management through soil application (NMPS) } & \multicolumn{5}{|c|}{0.46} & \multicolumn{5}{|c|}{29} \\
\hline \multicolumn{2}{|c|}{ Nutrient management through water soluble foliar fertilizers (WSFF) } & \multicolumn{5}{|c|}{0.69} & \multicolumn{5}{|c|}{28} \\
\hline \multicolumn{2}{|c|}{ WSFF at same NMPS } & \multicolumn{5}{|c|}{1.38} & \multicolumn{5}{|c|}{55} \\
\hline \multicolumn{2}{|c|}{ NMPS at same or different WSFF } & \multicolumn{5}{|c|}{1.28} & \multicolumn{5}{|c|}{56} \\
\hline \multicolumn{2}{|c|}{$\mathrm{WSFF}_{0}-$ Control (No foliar application of water soluble foliar fertilizer) } & \multicolumn{10}{|c|}{$\begin{array}{l}\mathrm{WSFF}_{1}-\text { Foliar application of water soluble foliar 19:19:19 fertilizer }\left(19 \% \mathrm{~N}, 19 \% \mathrm{P}_{2} \mathrm{O}_{5}\right. \\
\left.\text { and } 19 \% \mathrm{~K}_{2} \mathrm{O}\right) @ 2 \% \text { at } 25 \text { and } 40 \text { days after sowing }\end{array}$} \\
\hline \multicolumn{2}{|c|}{$\begin{array}{l}\text { WSFF }_{2}-\text { Foliar application of water soluble foliar 17:44 fertilizer } \\
\qquad\left(17 \% \mathrm{~N} \text { and } 44 \% \mathrm{P}_{2} \mathrm{O}_{5}\right) @ 2 \% \text { at } 25 \text { and } 40 \text { days after sowing }\end{array}$} & & & \multicolumn{8}{|c|}{$\mathrm{WSFF}_{3}-$ Foliar application of urea fertilizer $(46 \% \mathrm{~N}) @ 2 \%$ at 25 and 40 days after sowing } \\
\hline \multicolumn{2}{|c|}{ DAS - Days after sowing } & & & \multicolumn{8}{|c|}{ NS - Non-significant } \\
\hline
\end{tabular}


These results are in accordance with the findings of Sagare et al., (1986), Reddy et al., (1992), Veerabhadrappa, (2003), Chandrashekaran (2004), Haseeb and Maqbool (2015) who opined that in addition to soil applied fertilizers foliar spray of nutrients resulted in higher seed yield in sunflower and groundnut.

\section{References}

Anonymous, 2016, State of Indian Agriculture 2016, Directorate of Economics and Statistics, Ministry of Agriculture and Farmers welfare, Department of Agriculture, Cooperation and Farmers Welfare, GOI. pp. 119.

Banerjee, H., Dutta, S. K., Pramanik, S. J., Ray, K., Phonglosa, A. and Bhattacharyya, K., 2014, Productivity and profitability of spring planted sunflower hybrid with nitrogen, phosphorus and potassium fertilizer. Annals Pl. Soil Res., 16(3): 250-256.

Biradar, D. P., Aladakatti, Y. R. and Basavanneppa, M. A., 2012, Enhancing the productivity and economic returns of field crops with balanced nutrient application through site specific nutrient management approach. Proc. $3^{\text {rd }}$ National Conference on Agroinformatics and Precision Agriculture 2012 (AIPA, 2012) 1-3 August 2012, Hyderabad India. p. $146^{-1} 51$.

Chandrashekaran, 2004, Effect of foliar application of DAP and $\mathrm{ZnSO}_{4}$ on growth and productivity of groundnut. Int. J. Agric. Sci., 4(2): 548-550.

Gebremedhin, T., Shanwad, U. K., Gebremedhin, W. and Rama, A., 2015, Efficacy of foliar nutrition on vegetative and reproductive growth of sunflower (Helianthus annuus L.) Global J. Sci. Frontier Res. Agric. Vet., 15(9): 37-41.

Gomez, K. A. and Gomez, A. A., 1984,
Statistical Procedures for Agricultural Research, $2^{\text {nd }}$ Edition, A WileyInternational Science Publ., New York (USA), pp. 125-130.

Haseeb, M. and Maqbool, N., 2015, Influence of foliar applied nitrogen on reproductive growth of sunflower (Helianthus annuus L.) under water stress. Agric. Sci., 6:1413-1420.

Hegde, D. M. and Sudhakarababu, S. N., 2009, Declining factor productivity and improving nutrient-use efficiency in oilseeds. Indian J. Agron., 54(1): 1-8.

Hemalatha, S., Praveen, R., Padmaja, J. and Suresh, K., 2013, An over view on role of phosphorus on growth, yield and quality of sunflower. Int. J. Applied Biol. Pharm. Technol., 4(3): 48-55.

Iqbal, J., Hussain, B., Saleem, M. F., Munir, M. A. and Aslam, M., 2008, Bioeconomics of autumn planted sunflower (Helianthus annuus L.) hybrids under different NPK applications. Pakistan J. Agric. Sci., 45(3): 19-24.

Jebarathnam, G. T. and Sumalatha, 2014, Integrated nutrient management in sunflower and apiary farming system. Int. Res. J. Chem., 7: 01-11.

Khaliq, T. A., Ahmad, A. Hussain and Ali, M. A., 2008, Impact of nitrogen rate on growth, yield and radiation use efficiency of maize under varying environments. Pakistan J. Agri. Sci., 45: 1-7.

Khaliq, T., Ahmad, A., Hussain, A. and Ali, M. A., 2009, Maize hybrids response to nitrogen rates at multiple locations in semi arid environment. Pakistan J. Bot., 41: 207-224.

Ramulu, N., Krishnamurthy, H. M., Jayadeva, M., Janardhan, C. and Ramachandra, C., 2011, Influence of different levels of nutrient on growth and yield on sunflower under irrigated conditions of eastern dry zone of Karnataka, India. Plant Archives, 11(2): 1025-1028. 
Reddy, S. Y., Husain, M. M., Kasturi, K., Reddy, B. B. and Reddy, G. V., 1992, Effect of soil and foliar application of nitrogen in sunflower under rainfed conditions. Indian J. Agron., 37(4): 863-865.

Sagare, B. N., Naphade, K. T. and Joshi, B. G., 1986, Effect of urea and DAP sprays on yield and nutrient uptake by sunflower. J. Maharashtra Agric. Univ., 11: 54-56.

Veerabhadrappa, B. H., 2003, Effect of foliar nutrition (N, P, K, Ca and $\mathrm{S}$ ) on growth and yield of groundnut grown in alfisols. J. Oilseeds Res., 9(2): 2003.

\section{How to cite this article:}

Ravishankar, G., Lokanath H. Malligawad and Desai, B.K. 2019. Growth and Yield of Sunflower as Influenced by Nutrient Management Practices. Int.J.Curr.Microbiol.App.Sci. 8(12): 2882-2893. doi: https://doi.org/10.20546/ijcmas.2019.812.335 